\title{
PRICING OF ASIAN-TYPE AND BASKET OPTIONS VIA BOUNDS*
}

\author{
A. NOVIKOV ${ }^{\dagger}$, S. ALEXANDER ${ }^{\ddagger}$, N. KORDZAKHIA ${ }^{\S}$, AND T. LING ${ }^{\ddagger}$
}

(Translated by the authors)

\begin{abstract}
This paper sets out to provide a general framework for the pricing of average-type options via lower and upper bounds. This class of options includes Asian, basket, and options on the volume-weighted average price. The use of lower and upper bounds is proposed in response to the inherent difficulty in finding analytical representations for the true price of these options and the requirement for numerical procedures to be fast and efficient. We demonstrate that in some cases lower bounds allow for the dimensionality of the problem to be reduced and that these methods provide reasonable approximations to the price of the option.
\end{abstract}

Key words. Asian options, basket options, lower and upper bounds, volume-weighted average prices (VWAP), Lévy processes

DOI. $10.1137 /$ S0040585X97T987995

1. Introduction. The problem of pricing and hedging Asian-type and basket exotic options for more than two underlying assets has attracted the considerable attention of many researchers, primarily due to the increasing demand from practitioners for the development of fast, accurate, and stable numerical procedures suitable for the calculation of prices of path-dependent options. We believe that methods based on exact lower and upper bounds meet these requirements.

One of the first developments in this direction for arithmetic Asian call options under geometric Brownian motion (gBm) was obtained in 1994 by Curran [9]. Curran's approximation relies on the use of the geometric average process as a conditioning variable. The method and corresponding numerical procedure remain widely popular among industry practitioners to this day. Since 1994 there have been many further advances, successively leading to more accurate bounds under more complex models for the underlying asset, such as geometric Lévy processes (gLp), stochastic volatility (SV), and others (see, e.g., the recent papers [19], [6] and references therein). Typically, lower bounds $(L B \mathrm{~s})$ obtained in these papers have sufficient accuracy to allow them to be used as approximations for option prices and their sensitivities to parameters. Contrary to traditional approaches, which are usually based on finite-difference schemes for solving related partial differential equations (PDEs), the $L B$ s can also be calculated for high-dimensional problems, within reasonable computational time, while PDE methods remain unsuitable.

In this paper we provide some extensions to the results on lower and upper bounds obtained in [18] and demonstrate their applicability to problems recently discussed in the literature.

${ }^{*}$ Received by the editors March 13, 2015; revised March 31, 2015. This work was supported by Russian Scientific Fund grant 14-21-00162, and by Australian Research Council grants DP150102758 and DP130103315. Originally published in the Russian journal Teoriya Veroyatnostei $i$ ee Primeneniya, 61 (2016), No. 1, pp. 53-68.

http://www.siam.org/journals/tvp/61-1/T98799.html

$\dagger$ School of Mathematical and Physical Sciences, University of Technology, Sydney, Australia, and Steklov Mathematical Institute, RAS, Moscow, Russia (Alex.Novikov@uts.edu.au).

${ }^{\ddagger}$ School of Mathematical and Physical Sciences, University of Technology, Sydney, Australia.

$\S$ Department of Statistics, Macquarie University, North Ride, Sydney, Australia (Nino. Kordzakhia@mq.edu.au). 
In section 3 we discuss the problem of pricing options on volume-weighted average prices (see related papers [24], [19], [6]) and develop a new model for the volume process (we call it the "Lévy-volume model"), which is a natural generalization of the "gamma-volume model" suggested in [6].

In section 4 we deal with the prices and sensitivities of Asian options under a geometric Lévy process framework. This problem has been approached by many authors (see, e.g., [1], [11], [16], [4]). Here we present some new theoretical results and their numerical implementation based on the use of Fourier methods.

In section 5 we suggest a new lower bound for prices of multidimensional baskets and provide comparisons with recent results obtained in [15].

2. Notation. A unified approach to deriving lower and upper bounds. We assume that the prices of underlying assets $S_{t}=\left(S_{t}^{(1)}, \ldots, S_{t}^{(M)}\right), t \leqq T$, are defined on the filtered probability space $\left(\Omega, \mathcal{F},\left\{\mathcal{F}_{t}\right\}_{t \geqq 0}, \mathbf{P}\right)$, with $T$ the maturity time of European-type options, and also assume that the process $e^{-R_{t}} S_{t}$ is a martingale with respect to the (risk-neutral) probability measure $\mathbf{P}$ and that $R_{t}$ is the accrued interest at time $t$. These are standard assumptions in mathematical finance which are implied by the no-arbitrage hypothesis under a no-dividend setting (see, e.g., [23] concerning other details of this approach). This may be easily extended to the case where dividends are paid.

We aim to obtain accurate bounds for prices of Asian-type and basket options which, in a most general form, can be written as

$$
C_{T}=\mathbf{E} e^{-R_{T}}\left(\int_{0}^{T}\left(Y_{u}-K\right) \mu(d u)\right)^{+}
$$

where $x^{+}=\max [x, 0], K$ is a constant ("strike"), $Y=\left(Y_{u}, 0 \leqq u \leqq T\right.$ ) is a random process adapted to the filtration, and $\mu(u)$ is a (generally speaking) random distribution on $[0, T]$. As examples, one may consider the following financial options which occur often in practice. By taking $Y_{u}=\sum_{i=1}^{N} w_{i} S_{u}^{(i)}$, with $w=\left(w_{i}, i=\right.$ $1, \ldots, N)$ a given vector of weights and concentrating $\mu(u)$ at the point $u=T$, we obtain the price of a standard basket-call option

$$
C_{T}=\mathbf{E} e^{-R_{T}}\left(\sum_{i=1}^{N} w_{i} S_{T}^{(i)}-K\right)^{+} ;
$$

by setting $\mu(d u)=d u / T$, we obtain the price of a continuously-monitored (CMO) Asian basket call; by taking $\mu(d u)=d V_{u} / V_{T}$ with $V_{u}$ the accumulated total traded volume at the moment $u$, we obtain the case of options on the volume-weighted average price (VWAP). The notation can also accommodate the case of discretely monitored options (DMO) on VWAP:

$$
C_{T}=\mathbf{E} e^{-R_{T}}\left(\frac{\sum_{t_{j} \leqq T} S_{t_{j}} U_{t_{j}}}{\sum_{t_{j} \leqq T} U_{t_{j}}}-K\right)^{+},
$$

where $U_{t_{j}}$ is the traded volume at the moment $t_{j}$.

We also use the following convenient notation:

$$
\langle H, \mu\rangle:=\int_{0}^{T} H_{u} \mu(d u), \quad H \in \mathcal{H}^{\mu},
$$


where we suppose that the integral is well defined (e.g., $\mathcal{H}^{\mu}:=L^{1}(\Omega \times[0, T], \mathcal{F} \otimes$ $\mathbf{B}[0, T], d \mathbf{P} \times d \mu) .^{1}$

Using this notation we can rewrite (1) in the more compact form

$$
C_{T}=\mathbf{E} e^{-R_{T}}\langle Y-K, \mu\rangle^{+}=\mathbf{E} e^{-R_{T}}(\langle Y, \mu\rangle-K)^{+} .
$$

The following result is, in fact, a generalization of Theorem 1 of [18], which was proved under slightly different settings and notation.

Let $\mathbf{I}\{A\}$ be the indicator of the set $A$.

Proposition 1. Let $z \in \mathbf{R}, Y \in \mathcal{H}^{\mu}, H \in \mathcal{H}^{\mu}$. Then

$$
\begin{aligned}
& C_{T}=\max _{z \in \mathbf{R}, H \in \mathcal{H}^{\mu}}\left\{\mathbf{E} e^{-R_{T}}\langle Y-K, \mu\rangle \mathbf{I}\{\langle H, \mu\rangle>z\}\right\}, \\
& C_{T}=\min _{H \in \mathcal{H}^{\mu}}\left\{\mathbf{E} e^{-R_{T}}\left\langle(Y-K+\langle H, \mu\rangle-H)^{+}, \mu\right\rangle\right\},
\end{aligned}
$$

where both the maximum and the minimum are attained by taking $z=K, H=Y$.

These relations can be used for obtaining lower and upper bounds for $C_{T}$ by choosing a suitable random variable $H$ (we call it a "proxy"; see examples in what follows).

There exist many other similar relations, e.g., with arbitrary random variables $X$ in (5) instead of $\langle H, \mu\rangle$, but we would like to stress here that the maximum and the minimum in Proposition 1 are both attained by setting $H=Y$. To some extent relation (6) resembles one of the famous dual representations for American options suggested by Rogers [21].

For completeness of the exposition we provide the proof of Proposition 1.

Proof. For any $z \in \mathbf{R}$ and $H \in \mathcal{H}^{\mu}$

$$
\langle Y-K, \mu\rangle^{+} \geqq\langle Y-K, \mu\rangle \mathbf{I}\{\langle H, \mu\rangle>z\} .
$$

Thus we obtain

$$
C_{T}=\mathbf{E} e^{-R_{T}}\langle Y-K, \mu\rangle^{+} \geqq \sup _{z \in \mathbf{R}, H \in \mathcal{H}^{\mu}}\left\{\mathbf{E} e^{-R_{T}}\langle Y-K, \mu\rangle \mathbf{I}\{\langle H, \mu\rangle>z\}\right\} .
$$

Since $\langle Y-K, \mu\rangle^{+}=\langle Y-K, \mu\rangle \mathbf{I}\{\langle Y, \mu\rangle>K\}$, the equality in (5) is attained when $z=K$ and $H=Y$.

To prove (6) we note that for any $H \in \mathcal{H}^{\mu}$

$$
\begin{aligned}
\langle Y-K, \mu\rangle^{+} & =\langle Y-K+\langle H, \mu\rangle-H, \mu\rangle^{+} \\
& \leqq\left\langle(Y-K+\langle H, \mu\rangle-H)^{+}, \mu\right\rangle,
\end{aligned}
$$

where the last inequality is due to convexity of the function $x^{+}$. This implies that

$$
C_{T} \leqq \inf _{H \in \mathcal{H}^{\mu}}\left\{\mathbf{E} e^{-R_{T}}\left\langle(Y-K+\langle H, \mu\rangle-H)^{+}, \mu\right\rangle\right\} .
$$

Note that when $H=Y$ we have

$$
\mathbf{E}\langle Y-K+\langle Y, \mu\rangle-Y, \mu\rangle^{+}=\mathbf{E}(\langle Y, \mu\rangle-K)^{+}=C_{T} .
$$

Hence the minimum in (6) is achieved when $H=Y$ and (4) does indeed hold. Thus the proof of Proposition 1 has been completed.

\footnotetext{
${ }^{1}$ This notation was suggested by a reviewer.
} 
For the remainder of the paper we shall consider only the case where $R_{t}=r t$ with $r$ a constant. The case of time-dependent deterministic interest rates can be easily adapted to our framework (see related examples in [18]).

Due to (5) from Proposition 1 we have that for any $H \in \mathcal{H}^{\mu}$

$$
C_{T} \geqq L B:=\sup _{z \in \mathbf{R}}\left\{\mathbf{E} e^{-r T}\langle Y-K, \mu\rangle I\{\langle H, \mu\rangle>z\}\right\} .
$$

The evaluation of the lower bound $L B$ is typically much simpler than that of the true price $C_{T}$ due to the fact that (8) does not contain the nonlinear function $x^{+}$. As a result, one may note that for the evaluation of $L B$ we need only the joint distribution of $\log \left(Y_{t}\right)$ and $\langle H, \mu\rangle$; see examples in sections 3,4 , and 5 .

By (6) from Proposition 1 we have for any $H \in \mathcal{H}^{\mu}$ and any $a \in \mathbf{R}$

$$
C_{T} \leqq e^{-r T} \mathbf{E}\left\langle(Y-K+a\langle H, \mu\rangle-a H)^{+}, \mu\right\rangle
$$

and hence

$$
C_{T} \leqq U B:=e^{-r T} \inf _{a \in \mathbf{R}}\left\{\mathbf{E}\left\langle(Y-K+a\langle H, \mu\rangle-a H)^{+}, \mu\right\rangle\right\} .
$$

The evaluation of $U B$ is easier when compared to that of $C_{T}$ but it is typically more time consuming than the evaluation of the $L B$ (8) because it does involve the nonlinear function $x^{+}$.

A discussion concerning reasonable choices of the proxy random process $H^{*}$ in (8) and (9) as it relates to the production of accurate bounds is presented in [18]. We would like to remind the reader that the maximum and the minimum in Proposition 1 are both attained by setting $H=Y$, and therefore, one could expect that if we have a "good" approximation $H^{*}$ for $Y$ (i.e., leading to accurate $L B$ in (8)), then choosing the same proxy $H^{*}$ in (9) will produce a "good" $U B$ in (9). This consideration is often supported by comparisons with results of Monte Carlo simulations (see, e.g., [18], [4]), but of course, it would be desirable to have an analytical estimate of the distance between $U B$ and $L B$. In most examples the accuracy of $L B$, when compared with Monte Carlo simulation, is so good that such $L B s$ can even be used to approximate sensitivities (Greeks) via numerical differentiation.

3. Lower bound for options on VWAP under Lévy-volume model. In this section we discuss only the one-dimensional case and set $Y=S=\left(S_{t}, 0 \leqq\right.$ $t \leqq T)$. We shall assume that the process $S$ and the accumulated traded volume $V=\left(V_{t}, 0 \leqq t \leqq T\right)$ are independent.

3.1. Lévy-volume model. In [19] we applied the method of matching moments and log-normal approximation for $\int_{0}^{T} S_{u} d V_{u}$ under the assumptions that $S$ is a geometric Brownian motion $(\mathrm{gBm})$ and that the accumulated volume process $V_{t}=\int_{0}^{t} U_{u}^{2} d u$, with $U=\left(U_{t}, 0 \leqq t \leqq T\right)$ a squared Ornstein-Uhlenbeck process.

The key point of our approach in [19] was the development of a technique for finding the function

$$
g=\left(g_{t}:=\mathbf{E} \frac{U_{t}^{2}}{V_{T}}, t \leqq T\right)
$$

The method of finding $g$ proposed in [19] is based on the formula

$$
g_{t}=-\left.\int_{0}^{\infty} \frac{\partial}{\partial z} \mathbf{E} e^{-z U_{t}^{2}-q V_{T}}\right|_{z=0} d q
$$


which, as a matter of fact, holds for any random variable $U_{t}$ and any positive random variable $V_{t}$. Relation (10) leads to analytical representations for moments of VWAP and bounds for $C_{T}$ (see details in [17], [18], [19]).

Here we discuss another model that we call a Lévy-volume model, which is a generalization of the gamma-volume model suggested by Buryak and Guo [6]. The basic assumption of our model is that the increments of accumulated trading volume are increments of a Lévy subordinator $V=\left(V_{t}, t \leqq T\right)$, i.e., a process $V_{t}$ with independent and stationary increments, $V_{0}=0, \mathbf{P}\left(V_{t}>0\right)=1$ for $t>0$. Under this model for any trading moments $t_{j}$ and $t_{j-1}$ we define increments of trading volumes as follows:

$$
U_{t_{j}}:=V_{t_{j}}-V_{t_{j-1}}, \quad 0=t_{0}<t_{1}<\cdots<t_{N}=T .
$$

Note that in [6] it was assumed that $V_{t}$ is a gamma-process with a marginal gammadensity function

$$
\mathbf{P}\left(V_{t} \in d x\right)=\beta(\beta x)^{\alpha t-1} \frac{e^{-\beta x} d x}{\Gamma(\alpha t)}, \quad \alpha>0, \quad \beta>0, \quad t>0, \quad x>0 .
$$

We stress here that, contrary to the model with a squared Ornstein-Uhlenbeck process in [19] and [17], the accumulated Lévy-volume process $V_{t}$ has independent increments, and apparently, this leads to a simplification in the calculation of lower bounds for DMO and CMO on VWAP options.

In the CMO version of the Lévy-volume model it is natural to set

$$
\mu(d u)=\frac{d V_{u}}{V_{T}}, \quad 0 \leqq u \leqq T,
$$

and in the DMO version we shall assume that $\mu(u)$ has atoms at trading times $\left\{t_{j}, j=\right.$ $1, \ldots, N\}$ and hence use (3) for finding $C_{T}$.

For further calculations the following simple fact plays an important role.

Proposition 2. Let $V_{t}$ be a subordinator. Then

$$
f(u):=\mathbf{E} \frac{V_{u}}{V_{T}}=\frac{u}{T}, \quad u \leqq T .
$$

The proof of this fact is elementary when $u / T=i / N$ is a rational number. Indeed, we can then write $V_{u}=\sum_{j=1}^{i} \xi_{j}$, where random variables $\xi_{j}:=V_{j T / N}-V_{(j-1) T / N}$ are independent and identically distributed. Since by distribution $\left(\xi_{j}, V_{T}\right)=\left(\xi_{1}, V_{T}\right)$, we have

$$
\mathbf{E} \frac{V_{u}}{V_{T}}=\sum_{j=1}^{i} \mathbf{E} \frac{\xi_{j}}{V_{T}}=i \mathbf{E} \frac{\xi_{1}}{V_{T}}, \quad i=1,2, \ldots, N
$$

For $i=N$ this implies that $N \mathbf{E}\left(\xi_{1} / V_{T}\right)=1$, and therefore, $i \mathbf{E}\left(\xi_{1} / V_{T}\right)=i / N=u / T$.

The case of arbitrary $u$ and $T$ can be treated with a limit procedure or with the help of formula (10) or with the following considerations. It is easy to see that $f(u):=\mathbf{E} V_{u} / V_{T}$ is a nondecreasing function, $f(0)=0, f(T)=1$, and for any $u \in[0, T], s \in[0, T]$ such that $u+s \in[0, T]$

$$
\begin{aligned}
f(u+s) & =\mathbf{E} \frac{V_{u}+V_{u+s}-V_{u}}{V_{T}}=f(u)+\mathbf{E} \frac{V_{u+s}-V_{u}}{V_{T}} \\
& =f(u)+f(s),
\end{aligned}
$$


where the last equality is due to stationarity and independence of increments of $V_{t}$. It is well known that the monotonic solution of the functional equation $f(u+s)=$ $f(u)+f(s)$ is a linear function, and since $f(0)=0, f(T)=1$, we have $f(u)=u / T$.

3.2. Lower bounds. First, we note that due to the convexity of the function $x^{+}$and the assumption of independence of $V$ and $S_{t}$ (the dynamics of $S_{t}$ have not yet been specified), we always have a lower bound of the form

$$
C_{T}:=e^{-r T} \mathbf{E}\langle S-K, \mu\rangle^{+} \geqq e^{-r T} \mathbf{E}\langle S-K, \mathbf{E} \mu\rangle^{+},
$$

where, due to Proposition 2 under the Lévy-volume model, we have for the CMO case $\mathbf{E} \mu(d u)=d u / T$. This means that we can obtain $L B s$ for options on VWAP via $L B s$ for ordinary Asian options.

We have already demonstrated how our technique works for deriving $L B s$ of both CMO and DMO for Asian options under a Gaussian framework in [18], but for completeness of the exposition here we demonstrate some steps of this technique.

To allow for the comparison of results we assume further, as in [6], that $S_{t}$ is a $\mathrm{gBm}$ process. Recall that under the risk-neutral measure we have the representation

$$
S_{t}=S_{0} \exp \left\{\left(r-\frac{\sigma^{2}}{2}\right) t+\sigma B_{t}\right\}
$$

where $B=\left(B_{t}, t \leqq T\right)$ is a standard Brownian motion and $\sigma$ is the volatility. (Note that a generalization to the case of geometric Lévy models can be carried out with a technique reported in section 4.) For the CMO case we suggest using $\langle\log (S), \mu\rangle$ as the proxy $\langle H, \mu\rangle$ in (5). Under the assumptions listed above this leads to the following representation for $L B$ in the CMO case:

$$
L B^{C}:=e^{-r T} S_{0} \sup _{z} \mathbf{E}\left(\int_{0}^{T} e^{\left(r-\sigma^{2} / 2\right) u+\sigma B_{u}} \frac{d u}{T}-K\right) \mathbf{I}\left\{\int_{0}^{T} B_{t} \frac{d t}{T}>z\right\} .
$$

Similarly, in the DMO case we use the proxy $\frac{1}{N} \sum_{j=1}^{N} B_{t_{j}}$, and thus we have

$$
\begin{aligned}
L B^{D}:=e^{-r T} S_{0} \sup _{z}\left(\sum_{j=1}^{N} \mathbf{E} e^{\left(r-\sigma^{2} / 2\right) t_{j}+\sigma B_{t_{j}}} \mathbf{I}\left\{\frac{1}{N} \sum_{j=1}^{N} B_{t_{j}}>z\right\} \frac{t_{j}-t_{j_{-1}}}{T}\right. \\
\left.-K \mathbf{P}\left(\frac{1}{N} \sum_{j=1}^{N} B_{t_{j}}>z\right)\right) .
\end{aligned}
$$

The expressions (13) and (14) have already been discussed in [18] under a setting that includes general Gaussian processes. In particular, using the Girsanov transformation in the case of $\mathrm{gBm}$ we have managed to make further simplifications to (13) and obtained the following lower bound for the CMO case:

$$
\begin{aligned}
L B^{C}=e^{-r T} \sup _{z}( & \int_{0}^{T} S_{0} e^{r u} \operatorname{erfc}\left(\frac{z-\sigma u(1-u /(2 T))}{\sqrt{2 T / 3}}\right) \frac{d u}{T} \\
& \left.-K \operatorname{erfc}\left(\frac{z}{\sqrt{2 T / 3}}\right)\right)
\end{aligned}
$$

Copyright $\odot$ by SIAM. Unauthorized reproduction of this article is prohibited. 
where $\operatorname{erfc}(x):=(2 / \sqrt{\pi}) \int_{x}^{\infty} e^{-t^{2}} d t$ and $x \in \mathbf{R}$. In the DMO case with $t_{i}=i T / N$, (14) can be reduced to

$$
\begin{gathered}
L B^{D}=\frac{e^{-r T}}{2 T N} \sup _{z}\left(\sum_{i=1}^{N} e^{r t_{i}} S_{0} \operatorname{erfc}\left(z-\sigma t_{i}\left(T-\frac{t_{i}}{2}+\frac{T}{2 N}\right)\right)\right. \\
\left.-K \operatorname{erfc}\left(z\left(2 D_{N}\right)^{-1 / 2}\right)\right),
\end{gathered}
$$

where $D_{N}=(T / 3)\left(1+3 /(2 N)+1 /\left(2 N^{2}\right)\right)($ see $[18])$.

3.3. Numerical results. Table 1 presents values of the lower bounds $L B^{D}(15)$ and the log-normal approximations LogNorm based on the approximation of variance for VWAP from [6, equation (5.5)]. Note that the value $3.0120^{*}$ is from [6, Table 3]. We also provide Monte Carlo estimates $(M C)$ based on $10^{7}$ trajectories for the case discussed in [6], i.e., for a Lévy-volume model generated by a gamma-process $V_{t}$ with parameters $\alpha=10, \beta=1$ in (11). Standard mean-square deviations of $M C$ estimates in Tables 1, 4, and 5 are denoted as $S E$.

As in [6] we set $S_{0}=100, K=100, r=0.05, N=80$, and $T=0.317$.

TABLE 1

Gamma-volume model.

\begin{tabular}{ccccc}
\hline$\sigma$ & $L B^{D}$ & LogNorm & $\mathrm{MC}$ & $\mathrm{SE}$ \\
\hline 0.2 & 3.0057 & $3.0120^{*}$ & 3.0056 & 0.0013 \\
0.4 & 5.5570 & 6.0170 & 5.5866 & 0.0027 \\
0.6 & 8.1130 & 8.5930 & 8.1542 & 0.0042 \\
0.8 & 10.6580 & 11.1680 & 10.7190 & 0.0059 \\
\hline
\end{tabular}

The results from Table 1 confirm our observation made in [19], [18], [17] that the log-normal approximation is not accurate enough when values of $\sigma$ (volatilities) are relatively large. Meanwhile the relative errors for $L B^{D}$ are always less than $0.5 \%$.

4. Asian options under Lévy process framework. In this section we discuss some examples of the numerical evaluation of lower bounds for Asian options on a onedimensional asset $S$. We shall assume that the process $S$ is modeled as a geometric Lévy process.

4.1. Bounds for options with fixed strikes. Let $Z:=\left(Z_{t}=\log \left(S_{t} / S_{0}\right), 0 \leqq\right.$ $t \leqq T$ ), and let the interest rate $r$ be constant. Assuming in (5) $Y=S$ and using the proxy $H=Z$ we obtain the following formula for Asian options:

$$
C_{T} \geqq L B^{A}:=e^{-r T} \sup _{z \in \mathbf{R}} \mathbf{E}\left\langle S_{0} e^{Z}-K, \mu\right\rangle \mathbf{I}\{\langle Z, \mu\rangle>z\} .
$$

Similarly, using the proxy $H=a Z, a \in \mathbf{R}$, in (6) we have

$$
C_{T} \leqq U B^{A}:=e^{-r T} \inf _{a \in \mathbf{R}} \mathbf{E}\left\langle\left(S_{0} e^{Z}-K+a\langle Z, \mu\rangle-a Z\right)^{+}, \mu\right\rangle .
$$

This upper bound has been investigated in [18] under the gBm framework. In this paper we shall consider several examples under the gLp framework and find the lower bound $L B^{A}$, which is typically more accurate and simpler to calculate than $U B^{A}$. 
4.2. Computing lower bounds under the Lévy process framework with Fourier transforms. We assume that $Z=\left(Z_{t}=\log \left(S_{t} / S_{0}\right), 0 \leqq t \leqq T\right)$ is a Lévy process. We also assume that the joint characteristic function of $Z_{t}$ and $\langle Z, \mu\rangle$,

$$
\varphi(\xi, \zeta ; t):=\mathbf{E} e^{i \xi Z_{t}+i \zeta\langle Z, \mu\rangle},
$$

is known in an explicit form (see examples in what follows). We assume also that there exists the density function $f_{\langle Z, \mu\rangle}(z)$, calculated as the inverse Fourier transform of $\varphi(0, \zeta ; T)$.

The lower bound $L B^{A}$ can be calculated in two steps. In the first step we find the value of $z=\underline{z}$ that gives the global maximum in (17); in the second step we use $\underline{z}$ to find the maximum that is $L B^{A}$.

The equation for $\underline{z}$ can be found in the following way. Differentiating over $z$ the expression under the symbol sup in (16) and equating to zero we have

$$
\frac{d}{d z} \int_{0}^{T} \mathbf{E} e^{Z_{u}} \mathbf{I}\{\langle Z, \mu\rangle>z\} \mu(d u)=\frac{K}{S_{0}} f_{\langle Z, \mu\rangle}(\underline{z}) .
$$

Then using Fourier techniques on the conditional expectation (in a way similar to [16]) and noting various symmetries in the integrand we obtain

$$
\frac{1}{\pi} \int_{0}^{T} \int_{0}^{\infty} \Re\left(\varphi(-i, \zeta ; u) e^{-i \zeta \underline{z}}\right) d \zeta \mu(d u)=\frac{K}{S_{0}} f_{\langle Z, \mu\rangle}(\underline{z}) .
$$

Having $\underline{z}$, the lower bound $L B^{A}$ can be reduced to the calculation of the following integral: $\alpha_{1}>0, \alpha_{2}<-1$, and $\beta<0$,

$$
\begin{aligned}
L B^{A}:=\frac{e^{-R_{T}} S_{0}}{2 \pi^{2}} \int_{0}^{T} \int_{-\infty}^{\infty} & \int_{0}^{\infty} \Re\left(\widehat{h}_{1}(\xi, \zeta ; \underline{z}) \varphi\left(-\xi+i \alpha_{1},-\zeta+i \beta ; u\right)\right. \\
& \left.+\widehat{h}_{2}(\xi, \zeta ; \underline{z}) \varphi\left(-\xi+i \alpha_{2},-\zeta+i \beta ; u\right)\right) d \xi d \zeta \mu(d u),
\end{aligned}
$$

where

$$
\begin{aligned}
& \widehat{h}_{1}(\xi, \zeta ; \underline{z})=\left(\frac{K}{\left(\alpha_{1}+i \xi\right) S_{0}}-\frac{1}{\alpha_{1}+1+i \xi}\right) \frac{e^{\underline{z}(\beta+i \zeta)}}{\beta+i \zeta} \\
& \widehat{h}_{2}(\xi, \zeta ; \underline{z})=\left(\frac{1}{\alpha_{2}+1+i \xi}-\frac{K}{\left(\alpha_{2}+i \xi\right) S_{0}}\right) \frac{e^{\underline{z}(\beta+i \zeta)}}{\beta+i \zeta} .
\end{aligned}
$$

The derivation of (18) can be done in two steps. First, using the relations (5) and (17) we obtain

$$
\begin{aligned}
L B^{A} & :=e^{-r T} \mathbf{E}\left\langle S_{0} e^{Z}-K, \mu\right\rangle \mathbf{I}\{\langle Z, \mu\rangle>\underline{z}\} \\
& =e^{-R_{T}} S_{0} \int_{0}^{T}\left(\Psi_{1}(u ; \underline{z})+\Psi_{2}(u ; \underline{z})\right) \mu(d u),
\end{aligned}
$$

where

$$
\begin{aligned}
& \Psi_{1}(u ; \underline{z}):=\int_{-\infty}^{\infty} \int_{-\infty}^{\infty}\left(\left(e^{x}-\frac{K}{S_{0}}\right) \mathbf{I}\{x<0, y>\underline{z}\}\right) f(x, y ; u) d x d y, \\
& \Psi_{2}(u ; \underline{z}):=\int_{-\infty}^{\infty} \int_{-\infty}^{\infty}\left(\left(e^{x}-\frac{K}{S_{0}}\right) \mathbf{I}\{x>0, y>\underline{z}\}\right) f(x, y ; u) d x d y,
\end{aligned}
$$

and $f(x, y ; t)$ is the joint density of $\left(Z_{t},\langle Z, \mu\rangle\right)$. Then for the second step we use exponential-damping (see [5]), which leads to (18). 
4.3. Numerical results. Tables 2 and 3 display the lower bounds and approximations for the sensitivity with respect to $S_{0}$ ("Delta"), respectively, for a variety of processes (variance-gamma (VG), normal-inverse-Gaussian (NIG), Merton jumpdiffusion (JD); see details in [4]). We assume $S_{0}=K=100$, the monitoring points are equally spaced, time $T=1$ is measured in years, and $N=\infty$ indicates a continuously monitored option (CMO). The Monte Carlo estimates $(M C)$ are based on $10^{7}$ trajectories.

TABLE 2

Lower bounds $\left(L B^{A}\right)$ and $M C$ results.

\begin{tabular}{lccc}
\hline$X$ process & $N$ & $\mathrm{MC}$ & $L B^{A}$ \\
\hline $\mathrm{VG}$ & 10 & 6.2150 & 6.2118 \\
NIG & 20 & 5.9855 & 5.9770 \\
Merton & $\infty$ & 5.7730 & 5.7634 \\
\hline
\end{tabular}

TABLE 3

Delta approximations and $M C$ results.

\begin{tabular}{lccc}
\hline$X$ process & $N$ & MC & Delta \\
\hline VG & 10 & 0.5943 & 0.5943 \\
NIG & 20 & 0.5912 & 0.5914 \\
Merton & $\infty$ & 0.6084 & 0.6082 \\
\hline
\end{tabular}

Comparison of the $L B$ results to $M C$ estimates shows that the lower bound is very accurate and for most purposes may be taken to be the actual price.

5. Lower bound for baskets options. In this section we assume that $S=$ $\left(S_{t}^{(i)}, i=1, \ldots, N, 0 \leqq t \leqq T\right)$ is a multidimensional $\mathrm{gBm}$ and $R_{t}=r T$.

5.1. Kirk-type approximation for basket-spread option. A basket-spread option price for $N$ assets is defined by the formula

$$
C_{T}=\mathbf{E}\left(e^{-r T} \sum_{i=1}^{M} S_{T}^{(i)}-e^{-r T} \sum_{i=M+1}^{N} S_{T}^{(i)}-e^{-r T} K\right)^{+},
$$

which is a particular case of (1).

The first analytical formula for $C_{T}$ was obtained in [14]) for the two-asset exchange option with $M=1, N=2$, and $K=0$ under gBm by making a reduction to the Black-Scholes formula (Margrabe's formula). As with Asian options, it is not yet possible to obtain an exact solution for this option under the Black-Scholes framework when $N \geqq 2$ and $K>0$, due to the fact that a linear combination of log-normal random variables still has a nonidentifiable distribution. These options are traded in a variety of markets and are useful for hedging portfolios of long and short positions in underlying assets (see [7]).

A number of different approaches to approximate the basket pricing problem are found in the literature and they may be classed as numerical, closed-form, or semi-closed-form (see, e.g., [13]). A popular closed-form approximation was described in [12] (Kirk's approximation).

Of particular interest is the recent paper [15], which extends Kirk's approximation to multi-asset baskets of $N>2$. We now briefly describe their extension of Kirk's method. 
Set $F_{t}=e^{-r t} \sum_{i=1}^{M} S_{t}^{(i)}$ and $P_{t}=e^{-r t} \sum_{i=M+1}^{N} S_{t}^{(i)}+K$. Since $S_{t}^{(i)}$ are gBm, the dynamics of $F_{t}$ and $P_{t}$ are given by

$$
\begin{gathered}
d F_{t}=\sum_{i=1}^{M} \sigma_{i} S_{t}^{(i)} d B_{t}^{(i)}=F_{t} \sum_{i=1}^{M} \sigma_{i} \frac{S_{t}^{(i)}}{F_{t}} d B_{t}^{(i)}, \quad F_{0}=\sum_{i=1}^{M} S_{0}^{(i)} \\
d P_{t}=\sum_{i=M+1}^{N} \sigma_{i} S_{T}^{(i)} d B_{t}^{(i)}=P_{t} \sum_{i=M+1}^{N} \sigma_{i} \frac{S_{t}^{(i)}}{P_{t}} d B_{t}^{(i)}, \quad P_{0}=\sum_{i=M+1}^{N} S_{0}^{(i)}+e^{-r T} K
\end{gathered}
$$

where $\sigma_{i}$ are the volatilities of $S_{t}^{(i)}$ and $B_{t}^{(i)}$ are correlated standard Brownian motions with correlation coefficients

$$
\rho_{i j}=\mathbf{E}\left(B_{1}^{(i)} B_{1}^{(j)}\right)
$$

Kirk's approximation, and its extension to the multidimensional case in [15], are based on an approximation of $\left(S_{t}^{(i)} / F_{t}, S_{t}^{(i)} / P_{t}\right)$ for $0 \leqq t \leqq T$ by corresponding constants $\left(S_{0}^{(i)} / F_{0}, S_{0}^{(i)} / P_{0}\right)$, so $F_{t}$ is then approximated by the process $\widetilde{F}_{t}$ with dynamics

$$
d \widetilde{F}_{t}=\widetilde{F}_{t} \sum_{i=1}^{M} \widetilde{\sigma}_{i} d B_{t}^{(i)}, \quad \widetilde{\sigma}_{i}=\sigma_{i} \frac{S_{0}^{(i)}}{F_{0}} .
$$

Similarly, we may approximate $P_{t}$ by $\widehat{P}_{t}$ with dynamics

$$
d \widehat{P}_{t}=\widehat{P}_{t} \sum_{i=M+1}^{N} \widehat{\sigma}_{i} d B_{t}^{(i)}, \quad \widehat{\sigma}_{i}=\sigma_{i} \frac{S_{0}^{(i)}}{P_{0}+K} .
$$

Using $\widetilde{F}_{T}$ and $\widehat{P}_{T}$ the option price (20) is then approximated by

$$
\mathbf{E}\left(\widetilde{F}_{T}-\widehat{P}_{T}\right)^{+},
$$

which is precisely of the form for which Margrabe's formula applies since $\widetilde{F}_{T}$ and $\widehat{P}_{T}$ are log-normal. As reported in [15], this heuristic method gives accurate results for a range of $N$ and $M$; however, there are no analytical formulas for the accuracy of the approximations made so far.

5.2. Lower bounds. We now apply the methods described above in section 2 to find a lower bound for the basket-spread option price. Using the random variable $X_{T}:=\left(\log \widetilde{F}_{T}-\log \widehat{P}_{T}\right)$ as our proxy in (8), we have the following lower bound:

$$
L B^{B C}:=\sup _{z \in \mathbf{R}}\left(\begin{array}{c}
\sum_{i=1}^{M} e^{-r T} \mathbf{E} \mathbf{I}\left\{X_{T}>z\right\} S_{T}^{(i)} \\
-\sum_{i=M+1}^{N} e^{-r T} \mathbf{E} \mathbf{I}\left\{X_{T}>z\right\} S_{T}^{(i)}-e^{-r T} K \mathbf{P}\left(X_{T}>z\right)
\end{array}\right) .
$$

Next note that

$$
X_{T}=\sum_{i=1}^{M} \widetilde{\sigma}_{i} B_{T}^{(i)}-\sum_{i=M+1}^{N} \widehat{\sigma}_{i} B_{T}^{(i)}+\text { const. }
$$

Copyright $@$ ( ) by SIAM. Unauthorized reproduction of this article is prohibited. 
is normally distributed, so $\mathbf{P}\left(X_{T}>z\right)$ is easy to compute for each $z$. In order to evaluate the expectations $\mathbf{E I}\left\{X_{T}>z\right\} S_{T}^{(i)}$ in (22) observe that for every $i \in$ $\{1, \ldots, N\}$

$$
\mathbf{E} \mathbf{I}\left\{X_{T}>z\right\} S_{T}^{(i)}=S_{0}^{(i)} \widetilde{\mathbf{P}}^{(i)}\left(X_{T}>z\right)
$$

where the measures $\widetilde{\mathbf{P}}^{(i)}$ are defined via the Girsanov transformation with the RadonNikodým derivative

$$
\frac{d \widetilde{\mathbf{P}}^{(i)}}{d \mathbf{P}}=\exp \left\{\sigma_{i} B_{T}^{(i)}-\frac{T \sigma_{i}^{2}}{2}\right\}
$$

It is known that the Girsanov transformation preserves covariances of Gaussian random processes (see, e.g., [20, Proposition 2]), and therefore, we have

$$
\widetilde{\mathbf{E}}^{(i)} \widetilde{B}_{1}^{(i)} \widetilde{B}_{1}^{(j)}=\rho_{i j}
$$

Observe also that under the measure $\widetilde{\mathbf{P}}^{(i)}$

$$
\left\{\widetilde{B}_{t}^{(j)}:=B_{t}^{(j)}-\rho_{i j} \sigma_{i} t, 0 \leqq t \leqq T, j=1,2, \ldots, M\right\}
$$

are standard Brownian motions. Thus, we can easily find $\widetilde{\mathbf{P}}^{(i)}\left(X_{T}>z\right)$ giving the lower bound

$$
\begin{aligned}
L B^{B C}=e^{-r T} \sup _{z \in \mathbf{R}}( & \sum_{i=1}^{M} S_{0}^{(i)} \widetilde{\mathbf{P}}^{(i)}\left(X_{T}>z\right) \\
& \left.-\sum_{i=M+1}^{N} S_{0}^{(i)} \widetilde{\mathbf{P}}^{(i)}\left(X_{T}>z\right)-K \mathbf{P}\left(X_{T}>z\right)\right),
\end{aligned}
$$

where $\widetilde{\mathbf{P}}^{(i)}\left(X_{T} \leqq z\right)$ and $\mathbf{P}\left(X_{T} \leqq z\right)$ are Gaussian distributions with known parameters.

5.3. Numerical results. The above considerations can be easily adapted to the case where each stock $S_{t}^{(i)}$ has a constant dividend denoted $q_{i}$; the formulas above still hold except we replace $S_{0}^{(i)}$ with $S_{0}^{(i)} e^{q_{i} T}$.

Tables 4 and 5 show results for 3 and 200 asset basket options with parameter values from [15] to allow for comparisons.

(1) For the 3 asset case the parameters are $N=3, M=0, T=1, \sigma_{1 / 2 / 3}=0.3$, $r=0.05, S_{0}=(60,50,40), q_{1 / 2 / 3}=0.03, \rho_{12}=0.7, \rho_{13}=0.5$, and $\rho_{23}=0.3$.

(2) The parameter values for the 200 asset case are $N=100, M=50, T=1$, $\sigma_{i \leqq M}=0.5, \sigma_{i>M}=0.3, r=0.05, S_{i \leqq M}=10, S_{i>M}=9, q_{i \leqq M}=0.05, q_{i>M}=0.03$, $\rho_{i \leqq M \cap j \leqq M}=0.6, \rho_{i \leqq M \cap j>M}=0.4$, and $\rho_{i} M \cap j>M=0.5$.

Both our lower bound and the Lau and Lo method give good results for these particular examples, though we note that our method is better for out-of-the-money options. Both methods cannot give the exact price, but with the use of lower bounds we always know which side of the true price we are on.

Copyright (c) by SIAM. Unauthorized reproduction of this article is prohibited. 
TABLE 4

Results for 3 asset basket for varying strike $K$.

\begin{tabular}{lcccc}
\hline $\mathrm{K}$ & $L B^{B C}$ & Lau and Lo & $\mathrm{MC}$ & $\mathrm{SE}$ \\
\hline 50.0 & 98.0054 & 98.0054 & 98.0235 & 0.0116 \\
100.0 & 50.9571 & 50.9654 & 50.968 & 0.0114 \\
150.0 & 15.7622 & 15.767 & 15.7787 & 0.008 \\
200.0 & 2.9862 & 2.9715 & 2.9999 & 0.0037 \\
250.0 & 0.4303 & 0.4222 & 0.4379 & 0.0014 \\
300.0 & 0.0551 & 0.0528 & 0.0565 & 0.0005 \\
\hline
\end{tabular}

TABLE 5

Results for 200 asset basket for varying strike $K$.

\begin{tabular}{lcccc}
\hline $\mathrm{K}$ & $L B^{B C}$ & Lau and Lo & $\mathrm{MC}$ & $\mathrm{SE}$ \\
\hline 0.0 & 143.937 & 143.937 & 144.023 & 0.0715 \\
50.0 & 119.833 & 119.834 & 119.9215 & 0.0671 \\
100.0 & 99.3342 & 99.3511 & 99.3813 & 0.0625 \\
150.0 & 82.0757 & 82.123 & 82.1487 & 0.058 \\
\hline
\end{tabular}

6. Conclusions. In this paper a framework for pricing average-type options via lower and upper bounds has been proposed.

New notation, which is flexible enough to consider a wide spectrum of options, is proposed. We gave numerical examples for VWAP options, Asian options in models with Lévy processes, and basket-spread options.

Acknowledgments. The authors thank Juri Hinz, Alex Buryak, and other participants of the working seminar at National Australia Bank for useful discussions of the presented results.

\section{REFERENCES}

[1] A. Albrecher and M. Predota, On Asian option pricing for NIG Lévy processes, J. Comput. Appl. Math., 72 (2004), pp. 153-168.

[2] A. Albrecher, The valuation of Asian options for market models of exponential Lévy type, in Proceedings of the 2nd Actuarial and Financial Mathematics Conference, Royal Flemish Academy of Belgium for Arts and Sciences, 2004, pp. 11-20.

[3] H. Albrecher, P. A. Mayer, And W. Schoutens, General lower bounds for arithmetic Asian option prices, Appl. Math. Finance, 15 (2008), pp. 123-149.

[4] S. Alexander, A. Novikov, and N. Kordzakhia, Bounds on prices for Asian options via Fourier methods, ANZIAM J., 57 (2016), pp. 299-318.

[5] K. Borovkov AND A. Novikov, On a new approach to calculating expectations for option pricing, J. Appl. Probab., 39 (2002), pp. 889-895.

[6] A. Buryak and I. Guo, Effective and simple VWAP options pricing model, Int. J. Theor. Appl. Finance, 17 (2014), 1450036.

[7] R. Carmona and V. Durrleman, Pricing and hedging spread options, SIAM Rev., 45 (2003), pp. 627-685.

[8] P. Carr and D. B. Madan, Option valuation using the fast Fourier transform, J. Comput. Finance, 2 (1999), pp. 63-73.

[9] M. CuRran, Valuing Asian and portfolio options by conditioning on the geometric mean price, Management Sci., 40 (1994), pp. 1705-1711.

[10] H. Funahashi and M. Kijima, A Unified Approach for the Pricing of Options Related to Averages, preprint, 2014.

[11] M. Forde AND A. JACQUiER, Robust approximations for pricing Asian options and volatility swaps under stochastic volatility, Appl. Math. Finance, 17 (2010), pp. 241-259.

[12] E. KIRK, Correlation in the energy markets, in Managing Energy Price Risk, Risk Publications, London, 1995, pp. 71-78. 
[13] M. Krekel, J. De Kock, R. Korn, And M. Tin-Kwai, An analysis of pricing methods for basket options, Wilmott Magazine, 3 (2004), pp. 82-89.

[14] W. Margrabe, The value of an option to exchange one asset for another, J. Finance, 33 (1978), pp. $177-186$.

[15] C. LAU AND C. Lo, The pricing of basket-spread options, Quantitative Finance, 14 (2014), pp. 1971-1982.

[16] D. Lemmens, L. Z. J. Liang, J. Tempere, and A. De Scheppe, Pricing bounds for discrete arithmetic Asian options under Lévy models, Phys. A, 389 (2010), pp. 5193-5207.

[17] T. G. Ling, Study of Some Functionals of Standard and Fractional Brownian Motions with Applications in Quantitative Finance and Statistics, Ph.D. thesis, University of Technology, Sydney, 2014.

[18] A. A. Novikov And N. E. Kordzakhia, Lower and upper bounds for prices of Asian-type options, Proc. Steklov Inst. Math., 287 (2014), pp. 225-231.

[19] A. Novikov, N. Kordzakhia, And T. Ling, Pricing of volume-weighted average options: Analytical approximations and numerical results, in Inspired by Finance: The Musiela Festschrift, Springer, Cham, 2014, pp. 461-474.

[20] A. Novikov, N. Kordzakhia, And T. Ling, On moments of Pitman estimators: The case of fractional Brownian motion, Theory Probab. Appl., 58 (2014), pp. 601-614.

[21] L. C. G. Rogers, Monte Carlo valuation of American options, Math. Finance, 12 (2002), pp. 271-286.

[22] S. Simon, M. J. Goovaerts, and J. Dhaene, An easy computable upper bound for the price of an arithmetic Asian option, Insurance Math. Econom., 26 (2000), pp. 175-183.

[23] A. N. Shiryaev, Essentials of Stochastic Finance. Facts, Models, Theory, Translated from the Russian by N. Kruzhilin, Adv. Ser. Stat. Sci. Appl. Probab. 3, World Scientific, River Edge, NJ, 1999.

[24] A. W. StACE, A moment matching approach to the valuation of a volume weighted average price option, Internat. J. Theoret. Appl. Finance, 10 (2007), pp. 95-110.

[25] G. W. P. Thompson, Fast Narrow Bounds on the Value of Asian Options, working paper, Centre for Financial Research, Judge Institute of Management Science, University of Cambridge, Cambridge, UK, 2000.

[26] M. Vanmaele, J. Deelstra, J. Linnev, and M. Dhaene, Goovaerts bounds for the price of discretely sampled arithmetic Asian options, J. Comput. Appl. Math., 185 (2006), pp. 5190 .

Copyright (c) by SIAM. Unauthorized reproduction of this article is prohibited. 